\title{
Seltene Folge einer Hyperemesis gravidarum
}

\begin{abstract}
Müssen sich Schwangere ständig übergeben, können die Elektrolyte entgleisen. Besonders gefürchtet ist die Hypokaliämie, die in diesem Beispielfall die Muskelkraft raubte.
\end{abstract}

—ine 20-Jährige stellte sich in der 20. Schwangerschaftswoche

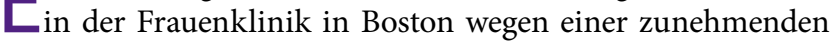
Schwäche in Armen und Beinen vor. In den Tagen zuvor war sie oft gestürzt, ihre Beine seien weggesackt. Schwindel oder Benommenheit verneinte sie. Seit Längerem litt sie unter Übelkeit und Erbrechen, weswegen sie in einem anderen Krankenhaus mehrmals Infusionen und Antiemetika erhielt. Sie konnte seit drei Tagen auch weder feste noch flüssige Nahrung bei sich behalten. Blutdruck, Körpertemperatur, Herz- und Atemfrequenz waren unauffällig. Die Eigenreflexe waren abgeschwächt; im EKG fiel eine U-Welle und ein verlängertes QTc-Intervall auf. Die labormedizinische Untersuchung bei Aufnahme bestätigte eine ausgeprägte Hypokaliämie $(1,8 \mathrm{mmol} / \mathrm{l})$ und Hypophosphatämie $(1,9 \mathrm{mmol} / \mathrm{l})$ sowie einen Anionen-Gap (27 mmol/l). Die Patientin wurde zunächst auf die Intensivstation verlegt und erhielt eine Volumen- und Elektrolytsubstitution mit $290 \mathrm{mEq}$ Kalium i.v. und $120 \mathrm{mEq}$ per os. Am Tag danach war die metabolische Azidose behoben, „was stark für eine Hungerketose bei ausgeprägter Dehydratation und metabolischer Entgleisung (Ketonkörper im Urin) sprach “, so die betreuenden Gynäkologen. Zwei Tage später klagte die Frau - nun auf der Normalstation - über zunehmende Muskelschmerzen, die Kreatinkinase-(CK)-Konzentration stieg auf $91.060 \mathrm{U} / \mathrm{l}$. Sie erhielt eine intensive Volumensubstitutionstherapie, anfangs $1.000 \mathrm{ml} / \mathrm{h}$, und parallel einen Phosphatbinder. Am Tag 12 hatte sich der CK-Wert mit $1.063 \mathrm{U} / \mathrm{l}$ deutlich verringert und es war kein Myoglobin mehr im Urin nachweisbar. Die Schwangere konnte entlassen werden, die Nierenfunktion wurde weiterhin engmaschig kontrolliert, die Kreatininwerte blieben stabil. Neben Folsäure erhielt sie ein Multivitaminpräparat mit Vitamin B6. Schon während des Krankenhausaufenthaltes verbesserte sich mit Normalisierung der Elektrolytkonzentrationen die Muskelschwäche.

Zeichen einer Rhabdomyolyse sind vor allem Muskelschmerzen und -schwäche, so die Studienautorin. Eine gefürchtete Komplikation der Rhabdomyolyse ist akutes Nierenversagen, dem man mit aggressiver Volumen- und Elektrolytsubstitution entgegenwirken kann. Neben Traumen, großer körperlicher Anstrengung, Muskelhypoxie und Medikamenten können auch Dehydratation sowie metabolische und Elektrolytimbalancen Muskelzellen zerstören. Die Bostoner Ärzte führten den Muskelzerfall auf die ausgeprägte Hypokaliämie zurück. Zwar fanden sie in der Literatur zwischen 1965 und 2015 keinen weiteren ähnlichen Fall, dennoch sollte unbedingt bedacht werden, dass die in Folge einer schweren Hyperemesis gravidarum auftretende Hypokaliämie auch eine Rhabdomyolyse verursachen kann. Dr.Dagmar Kraus

Lassey SC et al. Rhabdomyolysis After Hyperemesis Gravidarum. Obstet Gynecol 2016, online 6. Juni; doi: 10.1097/AOG.0000000000001418

\section{Hier steht eine Anzeige.}

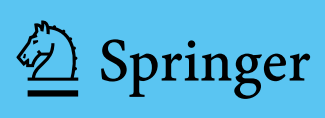

